If diagnosis means the identification of the disease from which the patient suffers it is only necessary to find tubercle bacilli to diagnose pulmonary tuberculosis. But it can also be defined as forming an appreciation of the patient, in the same sense as the commander of an army appreciates the military situation. It is then necessary to find out not only the identity of the infecting organism but also as much as possible about the pulmonary lesions and the qualities and environment of the patient. To identify pulmonary tuberculosis is relatively simple, but to appreciate the patient's condition is difficult; and it is with modern methods of doing it that this article is concerned.

\section{The Bacilli}

Tubercle bacilli can be sought for in sputum, or in bronchial secretions obtained by aspirating the fasting stomach contents, swabbing the pharynx and larynx or sucking out the bronchi at bronchoscopy. The 'laryngeal swab' would be more accurately called a ' cough swab' or ' pharyngeal swab,' for it is doubtful whether the larnyx is indeed swabbed in practice. But the results appear to be satisfactory, even using the simple technique of swabbing deeply behind the tongue and inducing a cough. Indeed, repeated swabbing is probably as effective as a single aspiration of the stomach. It is certainly far less uncomfortable for the patient and can easily be done on outpatients.

The Ziehl-Neelson method for staining tubercle bacilli in sputum has been largely replaced by the fluoresence technique in laboratories where large numbers of specimens are examined. Frequently, if the first one or two sputum samples examined by direct smear are negative, more and more are sent to the laboratory for the same examination. But it is unusual to find tubercle bacilli only in the last of a long series of direct smears. If the first specimens are negative it is better to concentrate and culture the sputum than to repeat direct examinations. Not only is there then a greatero chance of finding the bacilli, but also less work for 3 . the laboratory staff. Homogenisation and con-i. centration is probably most efficiently achieved by ${ }_{\omega}$ the Petroff method with 4 per cent. sodium hydroxide; but pancreatin has been used recently' and claimed to give better results. Löwenstein- $\overrightarrow{-0}$ Jensen is still the most efficient medium foro primary isolation. A positive result cannot be reported for about three weeks and a negative one for six. There have, therefore, been many. attempts to evolve more rapid procedures. Slideculture, in which growth in smears of sputumaise observed microscopically, has not been wides ${ }^{+}$ used as a routine. It is less efficient, technicafijo more difficult, and probably more dangerous? tos workers in busy laboratories. The smears have also been made from sputum homogenised byö pancreatin; it is claimed that they adhere betteo to the slide and give results only a little less accurate than the Löwenstein-Jensen methodЭ Liquid media give visible growth in a shortee time than solid media but cannot be used effectively. for primary isolation. They are employed is some methods for determining sensitivity to anti 3 . bacterial drugs. The procedure adopted in the M.R.C. Trials takes several weeks to completeThe results are, therefore, frequently of little value to the clinician. Even when the result is at last obtained, it is impossible to assess accuratel its significance to the individual patient, for tho bacilli tested are only a minute sample-probable not a representative one - of the whole bacillary population of the lungs. But a more rapid method would certainly be useful; and it is claimed thas sensitivity determinations by slide culture ar quick and reliable. Unfortunately, this and othe里 direct methods are least reliable when there are few bacilli in the sputum; and it is frequently in such eases that sensitivity tests are most wanted?

\section{The Lesions}

When the treatment of pulmonary tuberculos $\mathbb{\mathbb { Q }}$ 
consisted only of rest and inactive 'specifics' it did not matter much where or what the lesions were. But increasingly effective treatments have required increasingly precise diagnostic methods. The techniques of treatment and of radiographic diagnosis have evolved together, each stimulating the other. In some patients, however, it is still only necessary to know the approximate extent of the lesions; for, because of old age or the advanced character of the disease, this is all that is needed to treat them. In others, for instance where pulmonary resection is planned, it is essential to find out as much as possible about the extent, position and nature of the lesions. The circumstances of the individual patient determine how much investigation is necessary.

Although physical examination of the chest is certainly capable of discovering less about the pulmonary lesions than was at one time believed, it remains a necessary diagnostic procedure. For instance, persistent râles may be heard in a part of the lung radiographically normal. Or diminished breath sounds may indicate diminished aeration of a lobe when the radiograph shows little or no evidence of it.

\section{Radiology}

Progress in radiological diagnosis was for a long time held up by the difficulty of correlating the radiographic appearances with the pathological changes in the lung. This led to a loose terminology. Shadows were interpreted as ' infiltration,' ' fibrosis,' ' exudative,' and 'productive' with little justification. Pulmonary resection has removed some of the difficulties. It has become possible more frequently to check the radiographic interpretation with the morbid anatomy, especially in the earlier stages of the disease. The old terminology is now in many ways inadequate. 'Infiltration,' for instance, which neither describes the appearances nor in most instances the pathological condition, has been used for such varied lesions as scattered conglomerate tubercles, segmental consolidation, lobular pneumonia and bronchial abscesses. Unless the pathological state can be reasonably accurately inferred it would be better to use purely descriptive terms in reports on chest radiographs.

An approximate estimate of the extent of the lesions can be got from the postero-anterior film. The antero-posterior view may supplement this by making visible lesions hidden at the periphery of the lung field or in the upper zones. Lateral views are not very helpful, owing to the shadows from both lungs being superimposed and the shoulder girdle and spine obscuring the important upper and posterior parts of the lungs. Anteroposterior tomographs help considerably in explor- ing the apex of the lung and lateral tomographs the lower parts of the left lung that are obscured by the heart shadow in the postero-anterior film.

The distribution of the lesions can be investigated by lateral radiographs, but again they are of relatively little help if accurate localization is required. Nor are antero-posterior tomographs. Lateral tomographs give much more information. The lesions can almost always be located accurately within the lobes by means of the shadows of the fissures and the large pulmonary vessels. Localization to segments is still unfortunately not very reliable by any radiological technique. If there is much distortion of the normal gross anatomy it may be worth while outlining the bronchi with water-soluble radio-opaque material. This disappears rapidly from the bronchi and does not obscure the later radiographic appearances. But it may cause mild transient fever and some preparations are more irritating to the bronchi than oily media, requiring more thorough and extensive local anaesthesia. There is also the possibility of spreading the tuberculosis; but the danger appears slight. However, bronchograms seem in general to give no more information about the distribution of the lesions than do lateral tomographs and are certainly less comfortable for the patient.

Precise localization is increasingly important now that the first somewhat uncritical enthusiasm for excision is waning and conserving lung tissue is becoming as important as removing immediately harmless nodules. Unfortunately, localization is not always accurate. Moreover, even the most careful search by modern radiological methods sometimes fails to find some quite large lesions. It is even more difficult to infer the nature of them from the radiographic appearances. Lobar consolidation is relatively easy to detect, though the nature of the consolidation cannot be determined. Bronchopneumonia can be fairly confidentally diagnosed from the character of the coarse mottling. Airless and contracted lobes and segments usually give recognisable shadows; but atelectasis of smaller units of lung tissue may cause shadows indistinguishable from those of pneumonic areas. Calcified portions of tuberculous tissue usually show up well in tomographs, but many calcified lesions are quite indistinguishable radiographically. Fibrous lesions show fine linear opacities and there may be areas of increased radio-translucency with few vascular markings, indicating emphysematous patches. The prevalence of tuberculosis of the bronchi has been recognised in the last few years, largely as a result of examining resected specimens. Certain shadows strongly suggest bronchial lesions. There may be finger like or thick walled tubular opacities spreading out from 
the hilar shadows, or thick walled ring shadows sometimes with irregular inner margins, changing size relatively rapidly. Ring shadows can be caused by caseous lung lesions that have discharged into a bronchus, dilated bronchi with little or no alveolar involvement and emphysematous bullae; or there may be no cavity at all, the shadows being caused by differences in radiotranslucency within a solid tuberculous mass, or the superimposing of shadows caused by ribs, vessels, and fibrous strands. Tomography helps, but it is not infrequently impossible to do more than guess the nature of the 'cavities.' This also applies to circumscribed round shadows. Without evidence from the course of the disease and the appearances elsewhere in the lung patches of tuberculous pneumonia, granulomas, bronchial abscesses and filled in cavities cannot be distinguished. Although it is still seldom possible to infer the nature of the lung lesions from the radiographic appearances with confidence, modern techniques in conjunction with detailed examination of resected specimens have considerably increased the accuracy of diagnosis. Even a guess at the pathological appearances is better than such a misleading term as ' infiltration.'

Screening the chest does not help to determine the extent and distribution of the lesions. But it may help with their nature by demonstrating differences in radio-translucency of lobes or lungs and abnormal movements of the mediastinum when there is obstruction of major bronchi. It also gives information about the position and movements of the diaphragm, of use in assessing respiratory function.

Bronchography has little value in determining the nature of the lesions. It may show distortion of the bronchial tree or dilatation and irregularity of segmental bronchi. But frequently these can be accurately inferred from other evidence. Rarely it may reveal some unsuspected abnormality but this hardly justifies its use routinely, even before pulmonary resection. Occasionally strictures of the larger bronchi are clearly demonstrated and the information obtained, together with the bronchoscopic appearances, may then be valuable in planning treatment. Unfortunately, bronchography is not much help in investigating narrowing and occlusion of the smaller bronchi, for it is often impossible to decide whether an apparent blockage of a bronchus is due to disease of the walls, bronchial secretions or technical faults.

Progression of the lesions can be estimated only by serial radiographs. For accurate comparison it is essential to produce the same quality film each time. By variations of technique the shadows may be made to spread or recede, and new ones appear and disappear. Even with reasonably comparable films there are large difference $\overline{\bar{\delta}}$ between different observers' estimates of what is happening to the disease. One investigation, fo instance, showed that highly competent observers might disagree in almost a third of the serial: radiographs commonly seen in practice. More⿳亠丷厂二 over, a single observer might disagree wit himself in about $\frac{1}{5}$ th when the films were assesse a second time. The differences were by no means only between degrees of improvement og worsening. Where one observer recorded im provement of any degree the chances were that in about io per cent. of cases another would recor $\Phi$ deterioration. The subjective error in assessing changes in the lesions from radiographs has certainly been underestimated in the past.

\section{Bronchoscopy}

Knowledge of the gross appearances of the mait bronchi and a small part of the segmental bronchig is in many cases useful. It is unfortunate that ' tuberculous tracheo-bronchitis' has been given a name and hence become a 'diagnosis'; for it is almost always merely a part of the whole pul? monary disease. It only refers to disease of thos $\vec{e}$ portions of the bronchial tree that are seen throug the bronchoscope. Gross bronchitis of the seg $\Phi$ mental bronchus may be present without anyo bronchoscopic abnormality. Moreover, bronohy with florid microscopic bronchitis with recognisable mucosa can look healthy down the bronchoscope. Normal bronchoscopic appear ances, therefore, do not eliminate bronchias disease. But abnormal appearances help in treatment. It is, for instance, generally the practice to bronchoscope the patient before doing a thoracoplasty or resection. The result may not alter the decision to use a particular method of treatment, but it may change the timing of it, for tuberculous bronchitis frequently responds we to antibacterial drugs. It is also useful in patient with a persistently positive sputum withou evidence of cavities, atelectatic lobes in otherwise satisfactory artificial pneumothoraces, or with persistent one sided wheeze or other suggestives evidence of local abnormality of the major bronch? The risk of spreading the disease by bronchoscops is very slight ; but it is probably sufficient, together with the discomfort to the patient, to condemn 贷 as a routine diagnostic procedure.

\section{Lung Biopsy}

In some patients with diffuse lung changes i whom all other methods have failed to establish an adequate diagnosis, thoracotomy and lung biopsy may be useful. But there are probably very few instances indeed when histological evidence is so essential for rational treatment thof this procedure is justified. 


\section{The Patient}

The effect of the lesions on the patient is, of course, frequently obvious from the story of the illness. But there may only be slight weight loss and unusual tiredness, no symptoms at all, or only those that started when the patient learnt of the abnormal radiograph. Tiredness and loss of weight are also very common in non-tuberculous patients and they may well be unconnected with the lung lesions. Indeed, it is often impossible to judge from the story whether newly discovered lesions are having any effect on the patient at all. The general examination gives information about the patient's physique and appearance of wellbeing, and the presence of disease, tuberculous or non-tuberculous, in other systems. Examination of the chest gives, in addition to local signs of abnormality, evidence on the conformation and movenients of the chest wall, the aeration of the lungs and state of the bronchi. Measuring the vital capacity and maximum breathing capacity may help if some form of collapse or ablation treatment is being considered. The value of the more complicated measurements of respiratory function cannot yet be defined. It seems at present that they give no better estimate of the patient's ability to withstand surgical treatment than clinical observation and screening.

Fever is easily looked for, and taking the evening temperature every day for a week is a simple diagnostic method possibly too little used. The E.S.R. may help. Although the reading may be increased by non-tuberculous conditions or by technical errors, a single raised E.S.R. is suggestive and repeated abnormal readings are very good evidence that the pulmonary lesions are affecting the patient.

Serological tests are valueless in assessing the action of the lesions on the patient. The serum of patients with tuberculosis agglutinates red cells sensitized by contact with tuberculin much more frequently than that from non-tuberculous individuals. But although the incidence of positive agglutination tests seems to vary with the extent of the pulmonary disease it is not constantly related to the apparent 'activity' of the disease. The ' activity' of pulmonary tuberculosis is a very vague concept. 'Active' disease is not, as the term seems to imply, disease that is necessarily progressing or obviously affecting the patient. Activity ' is used in a special arbitrarily defined sense and is only useful for administration purposes. The idea behind it appears to be a mixture of the present action of the lesions and the probability of future progression. For clinical purposes these factors are better kept separate.

Diagnosis in the sense used here must, therefore, include an estimate of the probability that the disease will progress. The published results of investigations into the after history of patients, treated and untreated, together with the physician's own experience provide some guide. But the chances that the disease will some time progress depend very much on individual factors; and these can only be assessed from a thorough knowledge of the individual. This is important, for in many instances it is the probability of progression that decides the treatment rather than the present state of the lesions.

The usual process of history taking gives much information about the patient's personality and environment. But the more that can be found out the more effective the treatment can be. It is useful to know in some detail the whole circumstances of the patient in addition to his specific complaints. The type of accommodation and the facilities for maintaining a reasonable standard of hygiene, the personality of the husband and wife and the composition of the family, the financial position of the family and the patient's work, training, and ability to adapt himself to more suitable occupation will all have to be known. Facts and opinions supplied by the Almoner and Health Visitor supplement the physician's own observations and help him to form his appreciation of the patient.

The effective treatment of pulmonary tuberculosis requires, more than any other disease, intimate knowledge of the patient; for not only will advice be required for many years on such diverse subjects as choice of work, the use of leisure, marriage and conception, but the method of treatment may be more influenced by the type of patient than the type of lesion. It is possible to treat cases by conferences on radiographs; but patients need a physician.

\section{BIBLIOGRAPHY}

COCHRANE, A. L., GARLAND, L. H. (1952), ' Observer error in the interpretation of chest films,' Lancet, ii, 505 .

HINSON, K. F. W., JONES, A. R., CHAMBERLIN, J. A. (1952), The Middlebrook-Dubos reaction in relation to the clinical aspects of pulmonary tuberculosis,' Brit. $\mathcal{F}$. Tuberc., 46, 50.

McCLURE, D. M. (1953), "The development of fluorescence microscopy for tubercle bacilli and its use as an adjunct to histological routine,' $f$. Clin. Path., 6, 273.

MEDICAL RESEARCH COUNCIL' (I952), 'Cultivation of M. tuberculosis. Methods of homogenization,' Monthly Bull. of Minist. Hlth. Lond., II, 187.

MEDICAL RESEARCH CÖUNCIL (1953), 'Laboratory techniques for the determination of sensitivity of tubercle bacilli to isoniazed, streptomycin and P.A.S.', Lancet, ii, 213 .

McKECHNIE, J. K. (1953), 'Bronchography in Pulmonary tuberculosis,' Tubercle, 34, 271 .

RICE, H. M., KOWAN, F. C. (I953), ' The concentration, microculture and sensitivity testing of $M$. tuberculosis,' $\mathcal{F}$. Clin. Path.,

SIMÓN, $26 \mathrm{I}$. G. (1952), 'The lateral position on chest tomography,' ¥. Fac. Radiol, 4,6 .

SIMON, G. (1952), 'Tuberculous bronchitis and bronchiectasis,' F. Fac. Radiol, 5,33 .

YERUSHALMY, J., GARLAND, L. H., HARKNESS, J. T., HINSHAW,' H.' C., MILLER, E. K., SHIPMAN, S. J., ZWERLING, H. B.' (I95I), 'An' evaluation of the role of serial chest roentgenograms in estimating the progress of disease in patjents with pulmonary tuberculosis,' Amer. Rev. Tuberc., 64,225 . 